\title{
Contemporary knee arthroplasty: one fits all or time for diversity?
}

\author{
Johannes Beckmann $^{1} \cdot$ Malin Kristin Meier ${ }^{2} \mathbb{D} \cdot$ Christian Benignus $^{1} \cdot$ Andreas Hecker $^{2} \cdot$ Emmanuel Thienpont $^{3}$
}

Received: 12 April 2021 / Accepted: 1 July 2021 / Published online: 16 July 2021

(c) The Author(s) 2021

\begin{abstract}
Introduction Total knee arthroplasty (TKA) has historically been the preferred solution for any type of knee osteoarthritis, independently of the number of compartments involved. In these days of patient-specific medicine, mono-compartmental disease could also be approached with a more individualized treatment, such as partial knee arthroplasty (PKA). Off-theshelf (OTS) implants are often the compromise of averages and means of a limited series of anatomical parameters retrieved from patients and the pressure of cost control by limited inventory. Personalized medicine requires respect and interest for the individual shape and alignment of each patient.

Materials and methods A Pubmed and Google Scholar search were performed with the following terms: "patient-specific knee" and "arthroplasty" and "custom implant" and "total knee replacement" and "partial knee replacement" and "patellofemoral knee replacement" and "bicompartmental knee replacement". The full text of 90 articles was used to write this narrative review.

Results Unicondylar, patellofemoral and bicompartmental knee arthroplasty are successful treatment options, which can be considered over TKA for their bone and ligament sparing character and the superior functional outcome that can be obtained with resurfacing procedures. For TKA, where compromises dominate our choices, especially in patients with individual variations of their personal anatomy outside of the standard, a customized implant could be a preferable solution.

Conclusion TKA might not be the only solution for every patient with knee osteoarthritis, if personalized medicine wants to be offered. Patient-specific mono-compartmental resurfacing solutions, such as partial knee arthroplasty, can be part of the treatment options proposed by the expert surgeon. Customized implants and personalized alignment options have the potential to further improve clinical outcome by identifying the individual morphotype and respecting the diversity of the surgical population.
\end{abstract}

Keywords Partial knee arthroplasty · Unicondylar knee arthroplasty · Patellofemoral knee arthroplasty · Bicondylar knee arthroplasty $\cdot$ Customized knee arthroplasty

\section{Introduction}

Total knee arthroplasty (TKA) might no longer be the only suitable therapeutic option for each patient presenting with some type of knee osteoarthritis (OA). Usually, knee OA starts in one of the three knee compartments. Its

Malin Kristin Meier

malin.meier@icloud.com

1 Department of Endoprosthetics, Sportklinik Stuttgart, Stuttgart, Germany

2 Department of Orthopedic Surgery and Traumatology, Inselspital, Bern University Hospital, University of Bern, Bern, Switzerland

3 Cliniques Universitaires Saint-Luc, Bruxelles, Belgium aetiology is multifactorial and includes increased forces through the knee joint, either due to obesity [1] or because of alignment deviations [2-4]. Also anatomy modifying events such as previous trauma $[5,6]$ and total meniscectomy $[7,8]$ have been described as risk factors for the development of knee OA [1]. Depending on the degenerative status of the non-affected compartments and the philosophy of the treating surgeon, total knee arthroplasty (TKA) can be considered as an overtreatment. The times of a "one-fits-all approach", where all patients despite of age, gender, ethnicity and activity level, receive the same treatment are over. Besides the obvious differences in size between human beings, their demands and expectations might be different. The promising idea behind personalized medicine is to tailor the therapy for each individual 
person. While personalized medicine has already gained a foothold in other fields such as oncology, it is time to implement personalized medicine in orthopaedics as well.

Mono-compartimental OA can be an excellent example for this more individualized approach by treating the patient with a unicompartimental knee arthroplasty (UKA). The ligament and bone sparing character of this resurfacing procedure makes UKA an attractive solution, especially for young patients. In addition, better performance and less mortality after UKA have been reported [9-12]. However, the usage of UKA is still behind that of TKA. PKA reports lower survival in the different national registries and a three times higher revision rate. This more delicate surgery needs a more detailed understanding of indications and a more refined surgical technique, both asking for experience. However, the most frequent number of UKA done according to the UK register by each surgeon each year is 1 , followed by 2 [13]. The need for experience in every profession is evident and has been well described over the past years as a need for a higher percentage of UKAs within the surgeon's case mix [12, $14,15]$. Therefore, for the first time ever, a registry could show non-inferiority in revisions of UKA in hospitals with high usage [16].

But assistive technologies could be a proxy for surgical experience, which asks for time and patient volume. Besides choosing the optimal surgical approach for each individual patient, many technical factors can lead to lower failure rates, such as accuracy of cuts and component positioning, fixation techniques and advanced gap balancing. Because of the high variability in patients' individual anatomy, the use of an off-the-shelf implant with standard dimensions results in the adaption of the individual patient's anatomy to the implant, requiring excessive bone resections, the creation of symmetric gaps and the need to adapt the soft tissues to the structural modifications. Patients with an outlier anatomy will certainly benefit from a more customized knee arthroplasty.

The aim of this narrative review about personalized medicine in arthroplasty patients was to highlight the individual anatomic features that might lead to dissatisfaction, if they are modified outside the range of their limits because of surgical compromise. This can be because of a change in the constitutional alignment in all three planes, a lack of respect for the anthropometric features of each person or secondary symptoms, such as bone overload and subsidence, subtle or gross instabilities and overstuffing of different compartments.

In this narrative review, the authors will discuss indications for unicondylar, patellofemoral, bicompartmental, as well as customized total knee arthroplasty.

\section{Materials and methods}

A Pubmed and Google Scholar search was performed in January 2021 by two authors (JB \& MKM) with the following terms: "patient-specific knee" followed by adding "and arthroplasty" and "custom implant" and "total knee replacement" and "partial knee replacement" and "patellofemoral knee replacement" and "bicompartmental knee replacement". This lead to an initial 1029 papers that were found, reduced to 709 and 57 articles. The search for PKR and PFR lead 1085 studies to and bicompartmental knee replacement to 57 results. When in doubt to include a study a third author (ET) gave his advice and a majority choice was followed. After reading the abstracts and eliminating doubles, 90 papers remained and were considered useful for this narrative review. No risk of bias analysis was performed for the included studies.

\section{Results}

\section{Unicondylar knee arthroplasty}

\section{Indications}

The main indication for unicondylar knee arthroplasty (UKA) is mono-compartmental Kellgren-Lawrence stage IV osteoarthritis with anteromedial bone-on-bone contact [17]. The strict contraindications for UKA as described by Kozinn and Scott in 1989 [18] were attenuated over time with more evidence-based indications [19, 20]. The anterior cruciate ligament (ACL) needs to be functionally competent. In medial osteoarthritis (OA), an anterior location of the OA indicates that the ACL is intact. In contrast, a posterior location of OA indicates ACL deficiency [21]. Osteophytes within the other compartments can often be ignored if the joint space, and therefore the cartilage height, is completely intact in stress views or on ArthroCT [22]. A decreased joint space height of the other compartment is a contraindication for UKA, since this can lead to disease progression and revision [23]. For medial UKA, the medial collateral and anterior cruciate ligament have to be intact [19], the varus deformity has to be reducible so that on valgus stress radiographs a normal joint space height is visible. A coronal deformity of more than $15^{\circ}$, because of intra-articular wear, should also be considered a contraindication [24]. Medial patellofemoral OA without bone loss is no contraindication for isolated fixed or mobile bearing $[25,26]$, but it has been proven that lateral facet $\mathrm{OA}$ of the patella can lead to lower satisfaction rates and, therefore, might ultimately lead to revision [27]. The 
reason for this can easily be found in the coronal and axial realignment obtained by the intra-articular correction of the deformity during the resurfacing procedure of UKA. Flexion contracture over $10^{\circ}$ has been a contraindication for a long time, but a recent study showed that in a cohort with flexion contractures of up to $20^{\circ}$, improvement of functional scores was higher than after TKA, despite that some flexion contracture might remain after surgery. In this regard, more and better data are necessary for profound decision-making [28]. Obesity is no contraindication for performing UKA [19]. Even in patients with a BMI over 35 no inferior ten-year survival was found and especially this group showed the greatest improvement in Oxford Knee Scores (OKS) [29]. Chondrocalcinosis did not affect survival or functional outcome of UKA and is, therefore, not a contraindication, even if the other compartment is affected without cartilage loss [19, 30]. Age under 60 is associated with higher revision rates, but also with higher satisfaction rates compared to older patients. The main reason is thought to be a higher activity level of these patients. This has to be clearly discussed with the patient [24, 31]. Diffuse pain can occur in unicompartmental osteoarthritis due to diffuse synovitis and is not a contraindication for UKA [24]. Inflammatory disease or septic arthritis is clear and undebated contraindications.

The most important issue for good outcomes in a personalized arthroplasty program is to exclude patients with contraindications for that surgery [32].

\section{Outcome and survival comparing TKA and UKA}

A randomized controlled trial of 528 patients, randomly assigned to TKA or UKA, found no differences in survival, clinical outcomes, and revision rates at 5-year follow-up. However, length of hospital stay and effective costs were significantly lower in the UKA group. Patients answered more often yes to the question if they would undergo surgery again in the UKA group. A recent review of 60 studies, reported significantly better functional outcome scores for UKA, while pain did not differ between both groups. Furthermore, a higher mortality rate was reported for TKA $[12,33]$.

In contrast, a large registry study of more than 100000 matched patients comparing TKA and UKA found worse implant survival and a higher overall revision rate of UKA at 8 years [12]. However, registry-based data have the enormous bias of recording all implantations irrespective of the number of cases per surgeon.

A study of a high-volume centre with 2 years of followup, randomized patients to a simultaneous two team bilateral TKA or UKA procedure. The authors reported similar outcome regarding function, activity level and satisfaction, but also significant faster rehabilitation, less complications and less early revisions in UKA [34].

Lateral UKA is performed less frequently than medial UKA, but it is a good solution for isolated lateral disease. The reason for the lateral compartment, not to develop OA as often as the medial compartment, might be the biomechanical force distribution of 40-60\% with a higher load of the medial compartment [35]. Therefore, high-grade lateral OA is mostly posttraumatic due to fractures or after total meniscectomy. Implant survival has been reported comparable to medial UKA at 5, 10 and 15 years postoperatively [36]. No high-quality studies, i.e. randomized controlled trials, are available to compare medial to lateral UKA. The results of the many retrospective case series are partly controversial. Overall similar revision rates and subjective outcomes to medial UKA can be found [37]. Since the most reported complication leading to revision is dislocation in mobile bearings, fixed bearings should be the first choice for lateral UKA. The reason for higher bearing dislocation rates, in lateral than in medial UKA, is mostly seen in the naturally looser lateral flexion gap that can show inter-individual differences [37]. Mobile bearings showed biomechanical advantages, but these theoretical advantages were not reflected in the outcome of a randomized controlled trial with a 2-year follow-up [38]. The ideal indication for a lateral UKA, is a Kellgren-Lawrence stage IV osteoarthritis, often only seen on a Lyon schuss or Rosenbergs view, because the disease is posterolateral [39]. Restoration of the patient's individual anatomy is the goal of contemporary arthroplasty. Lateral UKA, as a resurfacing procedure, typically restores the patient's own morphotype, by the principle of epiphyseal resurfacing and, therefore, undercorrection, often leading to a better functional outcome [40].

The choice between UKA and TKA can often be a dilemma between function and implant survival as stated by Thienpont and Baldini in their editorial [41]. UKA's are revised threefold more than TKA's, but if we exclude the most frequent causes of revision such as aseptic loosening, polyethylene dislocation and infection, the other indications are often subjective and ad librum of the opinion of the revising surgeon. Registers show that disease progression, malalignment and unexplainable pain remain frequent causes for revision. If real bone to bone OA would be visualised in the contralateral compartment because of disease progression, two options exist. The most conventional one would be to revise to a TKA, but with the same success another UKA component could be added, respecting the central pivot and its stability [42].

UKA can also be a personalized solution for the elderly and more fragile patient. Since less blood loss [43] and less co-morbidity and mortality have been observed [12]. For those surgeons who believe they should identify the best patient-specific solution for each individual patient, UKA 
should certainly be one of their technical solution for monocompartmental OA [44].

The past of UKA has shown that the tibia is the difficulty to tackle with regard to implant fit. Conventional unicondylar systems usually offer only a medially mirrored model for the lateral compartment. The anatomical differences between the medial and lateral tibial plateau are not taken into account. The use of a custom UKA or a morphometric implant [45] can be the solution. Patient-specific instruments can easily help with accurate cuts, avoiding too deep posterior cuts or undercutting of the spine [46]. Patient-matched anatomical implants avoid over- and underhang, as well as subsidence or proximal tibial pain. A study reported significantly worse Oxford knee scores and pain scores 5 years postoperatively with tibial component overhang greater than $3 \mathrm{~mm}$ [47]. This study has emphasized the importance of respect for the tibial anatomy and the need to avoid impingement of the medial collateral ligament.

\section{Patellofemoral arthroplasty}

Isolated patellofemoral arthritis is found in $9 \%$ of all people over 40 years of age [48]. Women are affected more frequently and suffer from isolated patellofemoral arthritis in $24 \%$ of cases when gonarthrosis is present [49]. Proper patient selection is crucial. Hence, a detailed clinical examination, standard radiographs in three planes, a Rosenberg view and full leg standing radiographs are advised. MRI or ArthroCT to assess tibial tuberosity to trochlear groove (TTTG), as well as the quality of the cartilage in the other two compartments can also be recommended [22].

Patellofemoral joint arthroplasty (PFA) is the therapy of choice for isolated symptomatic osteoarthritis of the patellofemoral joint after unsuccessful conservative therapy when bone to bone OA Iwano IV is observed. The best indication is secondary OA due to patellofemoral dysplasia. Posttraumatic OA, after multiple surgeries of the patella or realignment procedures might be more difficult to treat with great patient satisfaction [50].

Contraindications include tibiofemoral instability, highgrade tibiofemoral arthritis (Kellgren-Lawrence 3 or 4), malalignment, infection, rheumatoid arthritis and ankylosis of the knee joint. The limits are an extension deficit $>10^{\circ}$, as well as a reduced flexion $\operatorname{arc}\left(<90^{\circ}\right)$ [51]. Following these strict indications, TKA can usually be avoided in isolated patellofemoral osteoarthritis, since young patients are mostly affected.

Two different systems are available for the trochlea: inlay or onlay prostheses. With inlay prostheses, only the defective cartilage of the trochlea is removed and milled out. This results in minimal bone loss and retention of the thickness of the trochlea, as the prosthesis is implanted at the level of the healthy cartilage. Reconstruction of the desired trochlea shape is achieved using implants with different degrees of curvature. Inlay prostheses are intended to prevent overstuffing of a normal trochlear anatomy [52]. However, in the case of rotational errors or axial deformities, additional procedures are often necessary to obtain accurate patellar tracking. Onlay prostheses, based on the concepts of TKA, require complete resection of the trochlea and are not dependent on the previous shape of the trochlea, so they can be used to treat and correct higher grades of dysplasia. Rotational defects, genu valgum or genu varum (within $3^{\circ}$ ) are not a contraindication and the alignment of the prosthesis can correct the patellofemoral tracking. There is increased bone loss and risk of overstuffing in comparison to inlay prostheses [53].

Inlay design showed a higher failure rate in the Australian registry compared with the onlay design, because of patellar maltracking, pain and clunck phenomenon [54]. Two studies from 2019, concerning the same inlay implant, obtained controversial results. While one showed promising results with a survival rate of $91 \%$ at 2 years and $83 \%$ at 5 years [55], the other found a high failure rate of these inlay prostheses [56]. Many patients had to be revised after 28 months and converted into an onlay prosthesis because of persistent pain and clunck phenomenon. Failure occurred mainly in patients with a high Insall-Salvati index, patella alta and craniolateral osteoarthritis. The choice for an inlay prosthesis is therefore described as a relative contraindication in these cases [56].

In comparison to TKA, PFA has been described superior in terms of knee function, postoperative activity scores and recovery time $[57,58]$. As mentioned above, the higher failure rates compared to TKA in registry data [13] needs to be levelled out with the more promising results of centres with a higher number of implantations. Ackroyd et al. examined 85 patients with 109 patellofemoral prostheses in a retrospective study and demonstrated a $95.8 \%$ survival rate after 5 years. Twenty-eight percent of patients had radiographic progression of osteoarthritis [59]. Another study showed that $75 \%$ of implanted first-generation prostheses were still functional after a mean follow-up of 10 years [60].

The main reason for revision surgery is obesity associated with coronal malalignment because this can lead to rapid progression of osteoarthritis in the tibiofemoral compartments. In these patients, primary implantation of a TKA should be considered what allows to correct their alignment issues [61]. The true indication for PFA is a patient with tibiofemoral hypertrophy of the cartilage in the presence of PFOA secondary to dysplasia [22].

A recent study showed that PFA is economically more favorable than TKA after 1 year of follow-up. The burden on health care systems can also be an important argument nowadays, within the therapeutic options. Further, good long-term survival of PFA has been reported with a survival 
ranging from 70 to $86 \%$ in a follow-up period ranging from 10 to 20 years $[62,63]$.

PFA seems to be preferable to TKA for well-selected patients with isolated patellofemoral arthritis. Third generation onlay designs allow the surgeon to change the dysplastic anatomy of the patient with recessing of the anterior aspect of the femur, a more open proximal trochlear angle and external rotation of the femoral component helping with patellar tracking and patellofemoral stability $[50,64]$.

Up to date, patient-specific PFA is not available. However, the low implantation rates of PFA and the low volume per surgeon, which is reflected in the controversial results of register studies and studies from high-volume centers, show that a patient-specific approach with patient-specific instruments for a higher surgical accuracy and patient-specific implants for an improved component fit, has great potential.

\section{Bicompartmental knee arthroplasty}

Thirty percent of patients treated with TKA have bicompartmental osteoarthritis [65]. The indication is often OA of the tibiofemoral compartment Kellgren-Lawrence stage IV combined with OA of the lateral patellar facet Iwano stage IV [66].

Today, bicompartmental solutions consist of a modular combination of UKA and PFA with two separate implants, because monobloc systems showed high complication [67] and failure rates [68]. This modular option requires a certain expertise of the surgeon when aligning the implants. Engh et al. compared 50 BKA with TKA in terms of restoring knee function. Differences in Knee Society Score and the Oxford Score were not significant. Functionally, there were initially advantages in favour of BKA, but this evened out after 2 years [69]. After a mean follow-up of 31 months, there was a postoperative improvement in Knee Society and Function Scores, KOOS, SF-12 and WOMAC, as well as in radiographic assessment and implant survival in another study. Of the 29 implanted bicompartmental prostheses, conversion to TKA was required in only one case after 3 years [70]. After a follow-up of $3.8 \pm 1.7$ years, there were better results for daily activities as well as better function in patients with BKA compared to TKA [71]. A literature review showed that BKA with modular components achieved good to excellent results after a follow-up of \pm 10 year. Especially, function and biomechanics are better compared to TKA [72]. However, BKA shows higher failure and revision rates compared to TKA [13]. Besides the low implantation number per surgeon and the complexity of the procedure, implant fit might also be a reason for this. Individual planning of the prostheses could provide an optimal fit and simplify the procedure. Implantation of medial or lateral UKA in combination with a PFA is possible. In a multicenter study, 78 patients received an individual custom
BKA. Improvements in knee mobility, as well as in knee scores (KSS and KOOS) were observed. Ninety-seven percent of implants survived after 2.6 years [73]. Ogura et al. could confirm these results, showing a survival rate of $98 \%$ after 2 years and $92 \%$ after 5 years. The WOMAC, VAS and SF-36 scores showed significant improvement and the patients were satisfied with the results [74]. The implantation of a custom BKA is promising. However, the data are manageable so far and longer follow-ups are necessary.

\section{Patient-specific TKA}

When patients need a TKA because of fixed deformities, important angular deviations [75] or the absence of ACL or PCL [21], the authors believe that the individual anatomy of the patient will be modified in many more ways than with the previous resurfacing type procedures. This has led to many different observations about potential causes for patient dissatisfaction observed in about $20-30 \%$ of the TKA population.

Shape mismatch has been considered a first cause of dissatisfaction and this has pushed the industry to develop different options for femoral and tibial components with gender specific implants (standard/narrow components) and addition of numerous (intermediate) sizes. Despite of this step forward with these morphometric implants, resulting in important inventory, imperfect component fit is still found when off-the-shelf implants are used. Several studies describe an association between persistent pain and worse functional outcome with component overhang [47, 76-79]. Femoral component overhang of more than $3 \mathrm{~mm}$ can be observed with a relatively high frequency, in particular in female patients. In these patients, the odds ratio for significant postoperative pain was doubled 2 years after surgery [78]. A CT study showed that up to $25 \%$ of the study cohort, especially patients with high anteroposterior and mediolateral dimensions, would have a mismatch between the bony anatomy and the femoral component of $3 \mathrm{~mm}$ if they would undergo surgery with a modern femoral component [80]. Intraoperatively, there are not many options as to choose a smaller implant size, if the component exceeds the bone if a standard OTS system is used. Downsizing may have a negative impact on the clinical outcome. Mediolaterally, cancellous bone that is not covered by the component, represents a risk for increased postoperative bleeding [81]. Anteroposteriorly, the posterior offset of the femur is decreased with an anterior referencing system, causing the tibial component to impinge with the femur earlier during roll back, which may result in decreased flexion capability [82]. With a posterior referencing system, this will lead to anterolateral notching depending of the external rotation [83].

A study on 24,042 CT data showed that $80 \%$ of the study cohort had a difference of more than $2 \mathrm{~mm}$ between the two 
posterior offsets [80]. However, most off-the-shelf systems do not take the difference between medial and lateral posterior condyle into account due to their mostly symmetrical design, which may lead to excessive medial bone resection, external rotation of the femoral component to achieve a balanced symmetric flexion gap and soft tissue releases, if mechanical alignment is used. Similar considerations must be made with the distal condylar offset to achieve a balanced extension gap [80]. Implants addressing this almost standard asymmetry were recently developed on femoral and corresponding inlay side. However, despite this modern and reasonable approach they again just offer a fixed amount neglecting the huge variability. Keeping in mind the findings of the huge database of $>24.000$ CT scans finding asymmetry as high as $8 \mathrm{~mm}[80]$.

Similarly, manufacturers introduced asymmetric designs to better replicate tibial anatomy. These designs have a fixed degree of asymmetry and a fixed ratio of mediolateral and anteroposterior widths, often increasing with implant size. However, another important CT study reported a high variability of tibial asymmetry that cannot be adequately addressed by off-the-shelf asymmetric designs [84]. Besides, some designs having symmetric anteroposterior dimensions. Using a symmetric component on an asymmetric tibia plateau results in either posterolateral overhang or posteromedial undercoverage, while the use of an asymmetric component on a symmetric tibial plateau leads to posterolateral undercoverage or posteromedial overhang. Furthermore, these compromises also affect and often negatively impact another important issue of tibial implant fit, namely implant rotation. Implant manufacturers continue to introduce more sizing options and alternative designs to address the anthropometric and gender differences. Narrower and wider femoral implants may address coverage issues and limitations in aspect ratio (AP/ML). Keep in mind the findings of a huge database of $>15.000$ CT scans that only $14 \%$ of tibiae exhibited symmetric $(<2 \mathrm{~mm}$ offset) lateral and medial plateaus, and $22 \%$ had an offset $>5 \mathrm{~mm}$ [84]. The problem for these new morphometric knee designs lies in the limited amount of patient-specific knee analyses that were performed to determine the shape of their future components. Furthermore, the shape of femur and tibia depends of the resection level, angles of correction and the rotation planned for that individual patient. More external rotation of the femur, with a symmetric implant, leads to either anterolateral notching or the use of a bigger AP size of femur [83]. A patientspecific rotation matches the original anatomy combined with a unique aspect ratio, guaranteeing perfect coverage. Already in 2014, an editorial entitled anthropometric measurements of the knee: time to make it fit [85] called for more implant variability. Last but not least, the slope of the tibia is different between the medial and lateral side with an intraindividual mean of $>2^{\circ}$ and differences as high as $>9^{\circ}[86]$.
So any tibial plateau design, with symmetrical polyethylene conformities, can only be an approximation of the individual anatomy.

With a customized total knee design, the component matches the patient's anatomy to ensure precise fit. In addition, the medial, lateral, and trochlear J-curves are anatomically reconstructed and the patient's native distal and posterior femoral offset is preserved. These design principles restore the relative position of ligament origin and insertion and therefore reduce the need for ligament balancing, but especially subtle instabilities in the three planes by modifications of the different ligament isometries.

Several studies reported better component fit of customized implants, including superior cortical bone coverage [87], less need for femoral and tibial bone resections [88] and optimal tibial rotation while maintaining proper bone coverage [89], compared to off-the-shelf implants.

Concerning knee kinematics, it has been reported that customized implants achieve kinematics that are more close to those of the native knee joint compared to off-the-shelf implants [90-92]. A study investigating contact stresses on the polyethyene using finite element models reported that customized implants had decreased contact stresses under gait cycle conditions [90].

Regarding clinical outcome, several studies reported on better function compared to off-the-shelf implants [74, 93, 94], resulting in higher patient satisfaction [74, 93]. Manufacturers of off-the-shelf implants added more implant sizes including narrow options to optimize the implant fit. It has been shown that this reduced the prevalence of femoral component overhang especially in female and Asian patients [95]. However, the costs of offering multiple implant sizes coming along with re-processing costs of multiple trays of instruments and implants [96] should be balanced against the initial higher costs of customized implants. Besides the re-processing costs, multiple trays may reduce the efficiency leading to an increased OR time and also longer turnover times between cases [96]. By reducing the amount of implants and instruments needed, customized implants can reduce turn over time. By reducing surgical steps, customized implants can reduce OR time, which is beneficial for the patient [96]. In addition it has been shown, that the use of customized implants can achieve savings in comparison with off-the-shelf procedures, driven by lower initial costs and lower postoperative spendings for inpatient services [97].

Even though it might be desirable to offer a customized implant to each patient to implement the approach of personalized medicine, there are patients that may benefit more from a customized implant than others. These patients have high anteroposterior or wide mediolateral dimensions, with differences in posterior and distal femoral offset and different degrees of tibial asymmetry. 
Despite of the yearlong focus on concepts like coverage and avoidance of overhang, other anatomic variances, such as overstuffing and three plane joint line changes can also affect function and patient satisfaction $[98,99]$. The optimal coronal alignment of TKA is still under debate and is reflected in the various alignment philosophies discussed today, such as mechanical alignment, anatomical alignment and kinematic alignment. In the past, surgeons aimed for a neutral mechanical alignment, because it was obtained most consistently with conventional instruments and it had been shown, that mechanical peak forces increase with incremental varus positioning of the tibial base plate [100]. However, opponents argue, that for a group of patients (i.e. patients with constitutional varus), neutral alignment is abnormal [101]. Alterations of the level and angle of the joint line can result in abnormal contact kinematics. Correction of coronal deformities requires soft tissue releases and the desire to avoid these has led to a growing interest in kinematic alignment in the recent years. Kinematic alignment aims to restore the individual pre-arthritic alignment [102]. However, complex and severe alignment deformities exist with intra- and extraarticular origins [21], and pre-arthritic alignment may differ from arthritic alignment. A recent review reported that current TKA alignment philosophies do not sufficiently address the high coronal variability in osteoarthritic knees [103]. This is further emphasized by a study who identified 162 different functional knee phenotypes in a CT study including 2764 osteoarthritic knees. They concluded that a more personalized alignment strategy in TKA is needed and that the challenge lies in identifying the optimal alignment strategy for the individual patient [104].

To allow a fully patient-specific approach, the individual knee anatomy including shape, alignment, kinematics and kinetics needs to be respected for all its diversity.

\section{Conclusion}

Dissatisfied patients and the continuous surgical compromises encountered during conventional TKA highlight the need for more personification in orthopaedics and more specifically in joint arthroplasty. A one-fits-all approach with conventional TKA is outdated. The disease should be treated with resurfacing procedures only where the disease actually is present. The combination of correct indication, customization of implants, preoperative 3D planning and patientspecific instruments has the great potential of improving patient satisfaction. Initially higher costs should be leveled with savings over time due to potential lower adverse event rates.
Funding Open Access funding provided by Universität Bern. No funds, grants, or other support was received.

\section{Declarations}

Conflict of interest The authors $\mathrm{AH}, \mathrm{CH}$ and MKM declare that they have no conflict of interest. The author JB declares that he receives honoraria from DePuy, Simth\&Nephew and Conformis (all outside the submitted work). The author ET declares that he receives consultancy fees from ConFormis, Convatec, Fidia, KCI, Lima, Zimmer Biomet, Royalties from Zimmer Biomet and Research grants from Zimmer Biomet. He is Board member of European Knee Society.

Ethical approval For this narrative review, no ethical approval was necessary.

Informed consent For this narrative review, no informed consent was necessary.

Open Access This article is licensed under a Creative Commons Attribution 4.0 International License, which permits use, sharing, adaptation, distribution and reproduction in any medium or format, as long as you give appropriate credit to the original author(s) and the source, provide a link to the Creative Commons licence, and indicate if changes were made. The images or other third party material in this article are included in the article's Creative Commons licence, unless indicated otherwise in a credit line to the material. If material is not included in the article's Creative Commons licence and your intended use is not permitted by statutory regulation or exceeds the permitted use, you will need to obtain permission directly from the copyright holder. To view a copy of this licence, visit http://creativecommons.org/licenses/by/4.0/.

\section{References}

1. Blagojevic M, Jinks C, Jeffery A, Jordan KP (2010) Risk factors for onset of osteoarthritis of the knee in older adults: a systematic review and meta-analysis. Osteoarthr Cartil 18:24-33

2. Felson DT, Niu J, Gross KD, Englund M, Sharma L, Cooke TDV, Guermazi A, Roemer FW, Segal N, Goggins JM, Lewis CE, Eaton C, Nevitt MC (2013) Valgus malalignment is a risk factor for lateral knee osteoarthritis incidence and progression: findings from the Multicenter Osteoarthritis Study and the osteoarthritis initiative. Arthritis Rheum 65:355-362

3. Sharma L, Chmiel JS, Almagor O, Felson D, Guermazi A, Roemer F, Lewis CE, Segal N, Torner J, Cooke TDV, Hietpas J, Lynch J, Nevitt M (2013) The role of varus and valgus alignment in the initial development of knee cartilage damage by MRI: the MOST study. Ann Rheum Dis 72:235-240

4. Sharma L, Song J, Dunlop D, Felson D, Lewis CE, Segal N, Torner J, Cooke TDV, Hietpas J, Lynch J, Nevitt M (2010) Varus and valgus alignment and incident and progressive knee osteoarthritis. Ann Rheum Dis 69:1940-1945

5. Driban JB, Eaton CB, Lo GH, Ward RJ, Lu B, McAlindon TE (2014) Knee injuries are associated with accelerated knee osteoarthritis progression: data from the osteoarthritis initiative. Arthritis Care Res (Hoboken) 66:1673-1679

6. Snoeker B, Turkiewicz A, Magnusson K, Frobell R, Yu D, Peat G, Englund M (2020) Risk of knee osteoarthritis after different types of knee injuries in young adults: a population-based cohort study. Br J Sports Med 54:725-730 
7. Englund M, Lohmander LS (2004) Risk factors for symptomatic knee osteoarthritis fifteen to twenty-two years after meniscectomy. Arthritis Rheum 50:2811-2819

8. Roos H, Laurén M, Adalberth T, Roos EM, Jonsson K, Lohmander LS (1998) Knee osteoarthritis after meniscectomy: prevalence of radiographic changes after twenty-one years, compared with matched controls. Arthritis Rheum 41:687-693

9. Drager J, Hart A, Khalil JA, Zukor DJ, Bergeron SG, Antoniou J (2016) Shorter hospital stay and lower 30-day readmission after unicondylar knee arthroplasty compared to total knee arthroplasty. J Arthroplasty 31:356-361

10. Fabre-Aubrespy M, Ollivier M, Pesenti S, Parratte S, Argenson J-N (2016) Unicompartmental knee arthroplasty in patients older than 75 results in better clinical outcomes and similar survivorship compared to total knee arthroplasty. A Matched Controlled Study. J Arthroplast 31:2668-2671

11. Hunt LP, Ben-Shlomo Y, Clark EM, Dieppe P, Judge A, MacGregor AJ, Tobias JH, Vernon K, Blom AW, National Joint Registry for England and Wales (2014) 45-day mortality after 467,779 knee replacements for osteoarthritis from the National Joint Registry for England and Wales: an observational study. Lancet 384:1429-1436

12. Liddle AD, Judge A, Pandit H, Murray DW (2014) Adverse outcomes after total and unicompartmental knee replacement in 101,330 matched patients: a study of data from the National Joint Registry for England and Wales. Lancet 384:1437-1445

13. National Joint Registry 15th Annual Report (2018) Healthcare Quality Improvement Partnership (HQIP). https://www.hqip.org.uk/resource/national-joint-regis try-15th-annual-report-2018/

14 Baker PN, Rushton S, Jameson SS, Reed M, Gregg P, Deehan DJ (2013) Patient satisfaction with total knee replacement cannot be predicted from pre-operative variables alone: a cohort study from the National Joint Registry for England and Wales. Bone Jt J 95-B:1359-1365

15. Murray DW, Parkinson RW (2018) Usage of unicompartmental knee arthroplasty. Bone Jt J 100:432-435

16. Endoprothesenregister Deutschland (EPRD) (2019) Jahresbericht 2019. https://www.eprd.de/de/ueber-uns/aktuelles/artikel/ eprd-jahresbericht-2019/

17. Kellgren JH, Lawrence JS (1957) Radiological assessment of osteo-arthrosis. Ann Rheum Dis 16:494-502

18. Kozinn SC, Scott R (1989) Unicondylar knee arthroplasty. J Bone Jt Surg Am 71:145-150

19. Beckmann J, Hirschmann MT, Matziolis G, Holz J, EisenhartRothe RV, Becher C (2021) Recommendations for unicondylar knee replacement in the course of time: a current inventory. Orthopade 50:104-111

20. Berend KR, Berend ME, Dalury DF, Argenson J-N, Dodd CA, Scott RD (2015) Consensus statement on indications and contraindications for medial unicompartmental knee arthroplasty. J Surg Orthop Adv 24:252-256

21. Thienpont E, Parvizi J (2016) A new classification for the varus knee. J Arthroplast 31:2156-2160

22. Thienpont E, Schwab P-E, Omoumi P (2014) Wear patterns in anteromedial osteoarthritis of the knee evaluated with CTarthrography. Knee 21:S15-S19

23. Waldstein W, Kasparek MF, Faschingbauer M, Windhager R, Boettner F (2017) Lateral-compartment osteophytes are not associated with lateral-compartment cartilage degeneration in arthritic varus knees. Clin Orthop Relat Res 475:1386-1392

24. Jennings JM, Kleeman-Forsthuber LT, Bolognesi MP (2019) Medial unicompartmental arthroplasty of the knee. J Am Acad Orthop Surg 27:166-176

25. Berger Y, Ftaita S, Thienpont E (2019) Does medial patellofemoral osteoarthritis influence outcome scores and risk of revision after fixed-bearing unicompartmental knee arthroplasty? Clin Orthop Relat Res 477:2041-2047

26. Kang SN, Smith TO, Sprenger De Rover WB, Walton NP (2011) Pre-operative patellofemoral degenerative changes do not affect the outcome after medial Oxford unicompartmental knee replacement: a report from an independent centre. J Bone Jt Surg Br 93:476-478

27 Konan S, Haddad FS (2016) Does location of patellofemoral chondral lesion influence outcome after Oxford medial compartmental knee arthroplasty? Bone Jt J 98-B:11-15

28. Purcell RL, Cody JP, Ammeen DJ, Goyal N, Engh GA (2018) Elimination of preoperative flexion contracture as a contraindication for unicompartmental knee arthroplasty. J Am Acad Orthop Surg 26:e158-e163

29. Molloy J, Kennedy J, Jenkins C, Mellon S, Dodd C, Murray D (2019) Obesity should not be considered a contraindication to medial Oxford UKA: long-term patient-reported outcomes and implant survival in 1000 knees. Knee Surg Sports Traumatol Arthrosc 27:2259-2265

30 Kumar V, Pandit HG, Liddle AD, Borror W, Jenkins C, Mellon SJ, Hamilton TW, Athanasou N, Dodd CAF, Murray DW (2017) Comparison of outcomes after UKA in patients with and without chondrocalcinosis: a matched cohort study. Knee Surg Sports Traumatol Arthrosc 25:319-324

31. Rodríguez-Merchán EC, Gómez-Cardero P (2018) Unicompartmental knee arthroplasty: current indications, technical issues and results. EFORT Open Rev 3:363-373

32. Willis-Owen CA, Brust K, Alsop H, Miraldo M, Cobb JP (2009) Unicondylar knee arthroplasty in the UK National Health Service: an analysis of candidacy, outcome and cost efficacy. Knee $16: 473-478$

33. Wilson HA, Middleton R, Abram SGF, Smith S, Alvand A, Jackson WF, Bottomley N, Hopewell S, Price AJ (2019) Patient relevant outcomes of unicompartmental versus total knee replacement: systematic review and meta-analysis. BMJ 364:1352

34. Kulshrestha V, Datta B, Kumar S, Mittal G (2017) Outcome of unicondylar knee arthroplasty vs total knee arthroplasty for early medial compartment arthritis: a randomized study. J Arthroplast 32:1460-1469

35. Werner FW, Ayers DC, Maletsky LP, Rullkoetter PJ (2005) The effect of valgus/varus malalignment on load distribution in total knee replacements. J Biomech 38:349-355

36. van der List JP, McDonald LS, Pearle AD (2015) Systematic review of medial versus lateral survivorship in unicompartmental knee arthroplasty. Knee 22:454-460

37 Bonanzinga T, Tanzi P, Altomare D, Dorotei A, Iacono F, Marcacci M (2020) High survivorship rate and good clinical outcomes at mid-term follow-up for lateral UKA: a systematic literature review. Knee Surg Sports Traumatol Arthrosc 3:1

38. Li MG, Yao F, Joss B, Ioppolo J, Nivbrant B, Wood D (2006) Mobile vs. fixed bearing unicondylar knee arthroplasty: a randomized study on short term clinical outcomes and knee kinematics. Knee 13:365-370

39. Merle-Vincent F, Vignon E, Brandt K, Piperno M, Coury-Lucas F, Conrozier T, Mathieu P, Hellio Le Graverand MP (2007) Superiority of the Lyon schuss view over the standing anteroposterior view for detecting joint space narrowing, especially in the lateral tibiofemoral compartment, in early knee osteoarthritis. Ann Rheum Dis 66:747-753

40. Thienpont E, Schwab PE, Cornu O, Bellemans J, Victor J (2017) Bone morphotypes of the varus and valgus knee. Arch Orthop Trauma Surg 137:393-400

41. Thienpont E, Baldini A (2014) Unicompartmental knee arthroplasty: function versus survivorship, do we have a clue? Knee 21:S1-S2 
42 Thienpont E (2017) Conversion of a unicompartmental knee arthroplasty to a total knee arthroplasty. Bone Jt J 99:65-69

43. Schwab P-E, Lavand'homme P, Yombi JC, Thienpont E (2015) Lower blood loss after unicompartmental than total knee arthroplasty. Knee Surg Sports Traumatol Arthrosc 23:3494-3500

44. Crawford DA, Berend KR, Thienpont E (2020) Unicompartmental Knee Arthroplasty: US and Global Perspectives. Orthop Clin North Am 51:147-159

45. Escudier J-C, Jacquet C, Flecher X, Parratte S, Ollivier M, Argenson J-N (2019) Better implant positioning and clinical outcomes with a morphometric unicompartmental knee arthroplasty. Results of a retrospective Matched-Controlled Study. J Arthroplast 34:2903-2908

46. Jones GG, Clarke S, Harris S, Jaere M, Aldalmani T, de Klee P, Cobb JP (2019) A novel patient-specific instrument design can deliver robotic level accuracy in unicompartmental knee arthroplasty. Knee 26:1421-1428

47. Chau R, Gulati A, Pandit H, Beard DJ, Price AJ, Dodd CAF, Gill HS, Murray DW (2009) Tibial component overhang following unicompartmental knee replacement-does it matter? Knee $16: 310-313$

48. Lonner JH (2010) Patellofemoral arthroplasty. Orthopedics 33:653

49 Davies AP, Vince AS, Shepstone L, Donell ST, Glasgow MM (2002) The radiologic prevalence of patellofemoral osteoarthritis. Clin Orthop Relat Res 402:206-212

50. Borus T, Brilhault J, Confalonieri N, Johnson D, Thienpont E (2014) Patellofemoral joint replacement, an evolving concept. Knee 21(Suppl 1):S47-50

51. Hurwit D, Strickland S (2020) Indications for patellofemoral arthroplasty in isolated patellofemoral arthritis. In: Dejour D, Zaffagnini S, Arendt EA, Sillanpää P, Dirisamer F (eds) Patellofemoral pain, instability, and arthritis: clinical presentation, imaging, and treatment. Springer, Berlin, pp 507-509

52. Davidson PA, Rivenburgh D (2008) Focal anatomic patellofemoral inlay resurfacing: theoretic basis, surgical technique, and case reports. Orthop Clin North Am 39:337-346 vii

53. Matz J, Howard JL, Morden DJ, MacDonald SJ, Teeter MG, Lanting BA (2017) Do changes in patellofemoral joint offset lead to adverse outcomes in total knee arthroplasty with patellar resurfacing? A radiographic review. J Arthroplast 32:783-787.e1

54. Australian Orthopaedic Association National Joint Replacement Registry Annual Reports (2016). https://aoanjrr.sahmri.com/ annual-reports-2016

55. Imhoff AB, Feucht MJ, Bartsch E, Cotic M, Pogorzelski J (2019) High patient satisfaction with significant improvement in knee function and pain relief after mid-term follow-up in patients with isolated patellofemoral inlay arthroplasty. Knee Surg Sports Traumatol Arthrosc 27:2251-2258

56. Beckmann J, Merz C, Huth J, Rath B, Schnurr C, Thienpont E (2019) Patella alta and patellar subluxation might lead to early failure with inlay patello-femoral joint arthroplasty. Knee Surg Sports Traumatol Arthrosc 27:685-691

57. Dahm DL, Al-Rayashi W, Dajani K, Shah JP, Levy BA, Stuart MJ (2010) Patellofemoral arthroplasty versus total knee arthroplasty in patients with isolated patellofemoral osteoarthritis. Am J Orthop 39:487-491

58. Odgaard A, Madsen F, Kristensen PW, Kappel A, Fabrin J (2018) The mark coventry award: patellofemoral arthroplasty results in better range of movement and early patient-reported outcomes than TKA. Clin Orthop Relat Res 476:87-100

59. Ackroyd CE, Newman JH, Evans R, Eldridge JDJ, Joslin CC (2007) The Avon patellofemoral arthroplasty. J Bone Jt Surg 89:6

60 Cartier P, Sanouiller J-L, Khefacha A (2005) Long-term results with the first patellofemoral prosthesis. Clin Orthop Relat Res. https://doi.org/10.1097/01.blo.0000171918.24998.d1
61. Bendixen NB, Eskelund PW, Odgaard A (2019) Failure modes of patellofemoral arthroplasty-registries vs. clinical studies: a systematic review. Acta Orthop 90:473-478

62. van Jonbergen H-PW, Werkman DM, Barnaart LF, van Kampen A (2010) Long-term outcomes of patellofemoral arthroplasty. J Arthroplast 25:1066-1071

63. Kooijman HJ, Driessen APPM, van Horn JR (2003) Long-term results of patellofemoral arthroplasty. A report of 56 arthroplasties with 17 years of follow-up. J Bone Jt Surg Br 85:836-840

64. Thienpont E, Lonner JH (2014) Coronal alignment of patellofemoral arthroplasty. Knee 21(Suppl 1):S51-57

65. Heekin RD, Fokin AA (2014) Incidence of bicompartmental osteoarthritis in patients undergoing total and unicompartmental knee arthroplasty: is the time ripe for a less radical treatment? J Knee Surg 27:77-81

66 Iwano T, Kurosawa H, Tokuyama H, Hoshikawa Y (1990) Roentgenographic and clinical findings of patellofemoral osteoarthrosis. With special reference to its relationship to femorotibial osteoarthrosis and etiologic factors. Clin Orthop Relat Res. https://doi.org/10.1097/00003086-199003000-00028

67. Tria AJ (2013) Bicompartmental knee arthroplasty: the clinical outcomes. Orthop Clin North Am 44:281-286 vii

68. Müller M, Matziolis G, Falk R, Hommel H (2012) The bicompartmental knee joint prosthesis journey Deuce: failure analysis and optimization strategies. Orthopade 41:894-904

69. Engh GA, Parks NL, Whitney CE (2014) A prospective randomized study of bicompartmental vs. total knee arthroplasty with functional testing and short term outcome. J Arthroplast 29:1790-1794

70. Kamath AF, Levack A, John T, Thomas BS, Lonner JH (2014) Minimum two-year outcomes of modular bicompartmental knee arthroplasty. J Arthroplast 29:75-79

71. Parratte S, Ollivier M, Opsomer G, Lunebourg A, Argenson $\mathrm{J}-\mathrm{N}$, Thienpont $\mathrm{E}$ (2015) Is knee function better with contemporary modular bicompartmental arthroplasty compared to total knee arthroplasty? Short-term outcomes of a prospective matched study including 68 cases. Orthop Traumatol Surg Res 101:547-552

72. Thienpont E, Price A (2013) Bicompartmental knee arthroplasty of the patellofemoral and medial compartments. Knee Surg Sports Traumatol Arthrosc 21:2523-2531

73. Beckmann J, Steinert AF, Huber B, Rudert M, Köck FX, Buhs M, Rolston L (2020) Customised bi-compartmental knee arthroplasty shows encouraging 3-year results: findings of a prospective, multicenter study. Knee Surg Sports Traumatol Arthrosc 28:1742-1749

74. Ogura T, Le K, Merkely G, Bryant T, Minas T (2019) A high level of satisfaction after bicompartmental individualized knee arthroplasty with patient-specific implants and instruments. Knee Surg Sports Traumatol Arthrosc 27:1487-1496

75. De Muylder J, Victor J, Cornu O, Kaminski L, Thienpont E (2015) Total knee arthroplasty in patients with substantial deformities using primary knee components. Knee Surg Sports Traumatol Arthrosc 23:3653-3659

76. Bonnin MP, Saffarini M, Shepherd D, Bossard N, Dantony E (2016) Oversizing the tibial component in TKAs: incidence, consequences and risk factors. Knee Surg Sports Traumatol Arthrosc 24:2532-2540

77. Bonnin MP, Schmidt A, Basiglini L, Bossard N, Dantony E (2013) Mediolateral oversizing influences pain, function, and flexion after TKA. Knee Surg Sports Traumatol Arthrosc 21:2314-2324

78. Mahoney OM, Kinsey T (2010) Overhang of the femoral component in total knee arthroplasty: risk factors and clinical consequences. J Bone Jt Surg Am 92:1115-1121 
79. Yan M, Wang J, Wang Y, Zhang J, Yue B, Zeng Y (2014) Gender-based differences in the dimensions of the femoral trochlea and condyles in the Chinese population: correlation to the risk of femoral component overhang. Knee 21:252-256

80 Meier M, Zingde S, Steinert A, Kurtz W, Koeck FX, Beckmann J (2018) What is the possible impact of high variability of distal femoral geometry on tKA? A CT data analysis of 24,042 knees. CORR. https://doi.org/10.1097/CORR.0000000000000611

81 Hitt K, Shurman JR, Greene K, McCarthy J, Moskal J, Hoeman T, Mont MA (2003) Anthropometric measurements of the human knee: correlation to the sizing of current knee arthroplasty systems. J Bone Jt Surg Am 85-A(Suppl 4):115-122

82. Bellemans J, Banks S, Victor J, Vandenneucker H, Moemans A (2002) Fluoroscopic analysis of the kinematics of deep flexion in total knee arthroplasty. Influence of posterior condylar offset. J Bone Jt Surg Br 84:50-53

83. Thienpont E, Bernardoni M, Goldberg T (2016) Anthropometric measurements of the femur change with component positioning in total knee arthroplasty. Knee 23:796-800

84 Meier M, Zingde S, Best R, Schroeder L, Beckmann J, Steinert AF (2019) High variability of proximal tibial asymmetry and slope: a CT data analysis of 15,807 osteoarthritic knees before TKA. Knee Surg Sports Traumatol Arthrosc. https://doi.org/ 10.1007/s00167-019-05728-4

85. Thienpont E, Becker R (2014) Anthropometric measurements of the knee: time to make it fit. Knee Surg Sports Traumatol Arthrosc 22:2889-2890

86. Meier M, Janssen D, Koeck FX, Thienpont E, Beckmann J, Best R (2021) Variations in medial and lateral slope and medial proximal Tibial angle. Knee Surg Sports Traumatol Arthrosc 29:939-946

87. Carpenter DP, Holmberg RR, Quartulli MJ, Barnes CL (2014) Tibial plateau coverage in UKA: a comparison of patient specific and off-the-shelf implants. J Arthroplast 29:1694-1698

88. Kurtz WB, Slamin JE, Doody SW (2016) Bone preservation in a novel patient specific total knee replacement. Reconstr Rev 6(1)

89. Schroeder L, Martin G (2019) in vivo tibial fit and rotational analysis of a customized, patient-specific TKA versus off-theshelf TKA. J Knee Surg 32:499-505

90. Koh Y-G, Lee J-A, Chung PK, Kang K-T (2019) Computational analysis of customized cruciate retaining total knee arthroplasty restoration of native knee joint biomechanics. Artif Organs 43:504-514

91. Patil S, Bunn A, Bugbee WD, Colwell CW, D'Lima DD (2015) Patient-specific implants with custom cutting blocks better approximate natural knee kinematics than standard TKA without custom cutting blocks. Knee 22:624-629

92. Zeller IM, Sharma A, Kurtz WB, Anderle MR, Komistek RD (2017) Customized versus patient-sized cruciate-retaining total knee arthroplasty: an in vivo kinematics study using mobile fluoroscopy. J Arthroplast 32:1344-1350

93. Reimann P, Brucker M, Arbab D, Lüring C (2019) Patient satisfaction-a comparison between patient-specific implants and conventional total knee arthroplasty. J Orthop 16:273-277
94. Wang H, Foster J, Franksen N, Estes J, Rolston L (2018) Gait analysis of patients with an off-the-shelf total knee replacement versus customized bi-compartmental knee replacement. Int Orthop (SICOT) 42:805-810

95. Sharma G, Liu D, Malhotra R, Zhou YX, Akagi M, Kim TK (2017) Availability of additional mediolateral implant option during total knee arthroplasty improves femoral component fit across ethnicities. JBJS Open Access. https://doi.org/10.2106/ JBJS.OA.16.00014

96. Sinha RK (2012) The use of customized TKA implants for increased efficiency in the OR. Curr Rev Musculoskelet Med 5:296-302

97. O'Connor MI, Blau BE (2019) The economic value of customized versus off-the-shelf knee implants in medicare fee-for-service beneficiaries. Am Health Drug Benefits 12:66-73

98 Hirschmann MT, Behrend H (2018) Functional knee phenotypes: a call for a more personalised and individualised approach to total knee arthroplasty? Knee Surg Sports Traumatol Arthrosc. https://doi.org/10.1007/s00167-018-4973-8

99. Hirschmann MT, Moser LB, Amsler F, Behrend H, Leclerq V, Hess S (2019) Functional knee phenotypes: a novel classification for phenotyping the coronal lower limb alignment based on the native alignment in young non-osteoarthritic patients. Knee Surg Sports Traumatol Arthrosc 27:1394-1402

100. Halder A, Kutzner I, Graichen F, Heinlein B, Beier A, Bergmann $\mathrm{G}$ (2012) Influence of limb alignment on mediolateral loading in total knee replacement: in vivo measurements in five patients. $\mathbf{J}$ Bone Jt Surg Am 94:1023-1029

101. Bellemans J, Colyn W, Vandenneucker H, Victor J (2012) The Chitranjan Ranawat award: is neutral mechanical alignment normal for all patients? The concept of constitutional varus. Clin Orthop Relat Res 470:45-53

102. Nisar S, Palan J, Rivière C, Emerton M, Pandit H (2020) Kinematic alignment in total knee arthroplasty. EFORT Open Rev 5:380-390

103. Hess S, Moser LB, Amsler F, Behrend H, Hirschmann MT (2019) Highly variable coronal tibial and femoral alignment in osteoarthritic knees: a systematic review. Knee Surg Sports Traumatol Arthrosc 27:1368-1377

104. Moser L, Hess S, Behrend H, Hirschmann M (2020) Variability of functional knee phenotypes in osteoarthritic knees shows that a more personalized approach in TKA is needed. Orthop J Sports Med 8:2325967120S00300

Publisher's Note Springer Nature remains neutral with regard to jurisdictional claims in published maps and institutional affiliations. 